\title{
[ARTIGO RETRATADO] Consumo máximo de oxigênio em atletas de futebol por meio de diferentes métodos
}

\author{
Fabrício Azevedo Voltarelli(I); Carlos Alexandre Fett(I); Christianne de Faria \\ Coelho(I); Fabrício Cesar de Paula Ravagnani(II)
}

(I) Universidade Federal de Mato Grosso (UFMT) - Departamento de Educação Física - Núcleo de Aptidão Física, Metabolismo e Saúde (NAFiMeS) - Cuiabá - MT

(II)Centro Universitário de Várzea Grande (UNIVAG) - Várzea Grande - MT

O motivo da retratação não adentra os motivos de plágio, uma vez que o texto foi confeccionado por todos os autores e não configura cópia de outro pré-existente; o mesmo foi baseado nos dados numéricos supostamente obtidos por meio de coletas de campo realizada, no ano de 2008, por graduandos os quais pertenciam a um grupo de pesquisa sob minha coordenação. Tal grupo de pesquisa não se formou na UFMT, uma vez que em 2008, ano das coletas, eu não havia, ainda, sido aprovado em concurso público e, portanto, trabalhava em uma Universidade Particular. Quando em Cuiabá, acessamos os dados (já tabulados, com estatística feita) e escrevemos o manuscrito, confiando plenamente nos dados numéricos. Vale ressaltar que não participei, in loco, das coletas efetuadas pelos alunos.

Devo destacar que os nomes dos alunos não foram incluídos no artigo submetido e aprovado pela RBME, pois, quando da oportunidade, os alunos não mostraram interesse na inserção, alegando que não pretendiam seguir carreira acadêmica e que nada somaria em suas vidas um artigo publicado. Dessa forma, fiz o convite aos outros colegas co-autores para efetuarmos a porção intelectual do trabalho.

Ano passado, requisitei a esses alunos, com os quais não mais mantenho contato presencial, os dados brutos dos valores contidos no artigo, pois, agregados aos mesmos, estavam outros dados (não utilizados) que usaríamos em outros trabalhos científicos visando publicação. No entanto, os ex-alunos disseram que os dados brutos não mais existiam, fato que causou estranheza e desconfiança de nossa parte, sendo que, a época, os dados haviam sido gravados no computador da Instituição. Como teriam sido apagado então? Essa foi a dúvida que pairou. Portanto, frente à essa situação, passei a não acreditar nos dados de nosso artigo publicado na RBME, uma vez que levantou-se a hipótese de que esses dados podem ter sido inventados. Tal prática não condiz com a ética e com os preceitos de nosso atual grupo. Dessa forma, para não permanecer sempre com dúvidas em relação aos dados contidos no artigo, decidi fazer a retratação e pedir a exclusão do artigo, pois, assim, acredito que dou minha parcela de contribuição para o meio acadêmico e, pricipalmente, para a RBME, uma vez que tal periódico é de elevada expressão acadêmica e respeitada por todos.

Atenciosamente,

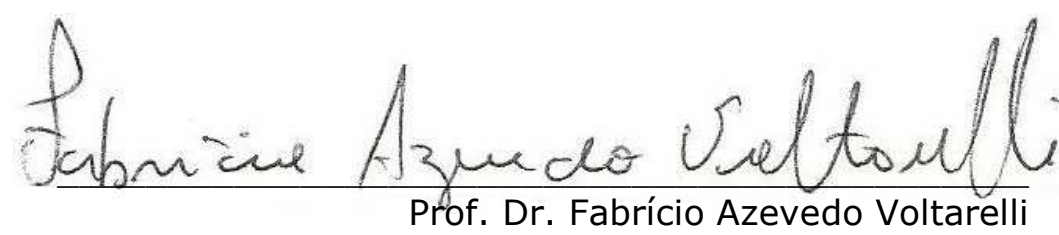

(Solicitante) 


\title{
Consumo Máximo de Oxigênio em Atletas de Futebol por Meio de Diferentes Métodos
}

\author{
Maximal Oxygen Consumption of Soccer Players by Different Methods
}

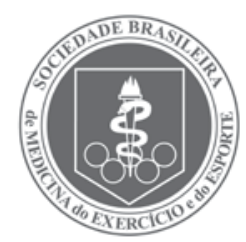

Artigo Original

Fabrício Azevedo Voltarelli ${ }^{1}$

Carlos Alexandre Fett ${ }^{1}$

Christianne de Faria Coelho

Fabrício Cesar de Paula Ravagnani ${ }^{2}$

1. Universidade Federal de Mato Grosso (UFMT) - Departamento de Educação Física - Núcleo de Aptidão Física, Metabolismo e Saúde (NAFiMeS) - Cuiabá -MT

2. Centro Universitário de Várzea

Grande (UNIVAG) - Várzea Grande - MT

\section{Correspondência:}

Universidade Federal de Mato Grosso (UFMT) - Departamento de Educação Física Faculdade de Educação Física (FEF) Avenida Fernando Corrêa da Costa, 2367 - 78060-900 - Cuiabá-MT E-mail: favoltarelli@ufmt.br

\section{RESUMO}

O presente estudo avaliou o consumo máximo de oxigênio $\left(\dot{\mathbf{W}^{2}}{ }_{2 m a ́ x}\right)$ de jogadores profissionais de futebol. Vinte jogadores de futebol $(24,12 \pm 0,81$ anos de idade) participaram do estudo Todos os sujeitos efetuaram dois testes máximos de campo: a) Yo-yo teste (T1) e b) Yo-yo teste intermitente (T2) usando ergoespirometria portátil. Além disso, dois testes máximos em esteira rolante foram realizados por melo de protocolos contínuos (T3) e intermitentes (T4). Todos os resultados foram expressos como média - desvio padrão. ANOVA de duas entradas para medidas repetidas foi usada para determinar possivive diferenças estatísticas $(p<0,05)$. 0 $\dot{\mathrm{V}}_{2 \text { máx }}\left(\mathrm{ml} \cdot \mathrm{kg}^{-1} \cdot \mathrm{min}^{-1}\right)$ estimado de $\mathrm{T1}(54,6 \pm 2,2)$ foi significativamente menor em relação aos testes $\mathrm{T2}(61,2$ $\pm 3,3)$, T3 $(62,2 \pm 4,3)$ e T4 $(63,7 \pm 4,5)$. Diferenças entre os protocolos de exercicios intermitentes (T2 e T4) contínuos (T1 e T3) foram também observadas. Tomados em conjunto, os resultados do presente estudo sugerem que o Yo-yo teste pode ser usado no programa de ireinamento, como uma alternativa mais barata, objetivando a determinação da capacidade aeróbia.

Palavras-chave: $\dot{\mathrm{V}}_{2 \text { máx }}$ testes de campo, testes laboratoriais

\section{ABSTRACT}

The present study aimed to evaluate the maximal oxygen uptake $\left(\dot{\mathrm{V}}_{\mathrm{O}_{\text {max }}}\right)$ in professional soccer players. Twenty soccer players (24.1 \pm 0.81 years oid) participated in of this study. All subjects performed two maximal field tests: a) Yo-Yo endurance test (T 1) and b) Yo-Yo intermittent endurance test (T2) using portable telemetric ergospirometry. In addition, two maximal exercise tests on the treadmill with continuous (T3) and intermittent (T4) protocols were employed. The $\dot{\mathrm{V}}_{2 \max }$ was assessed by direct ergospirometry in T3 and T4. All results were expressed as mean \pm standard deviation. Two-way ANOVA two way for repeated measurements was used to determine statistical differences $(p<0.05)$. The estimated $\dot{\mathrm{V}} \mathrm{O}_{2 \max }\left(\mathrm{ml}^{\mathrm{K}} \mathrm{\textrm {Kg } ^ { - 1 }} \cdot \mathrm{min}^{-1}\right)$ of the $\mathrm{T} 1$ test $(54.6$ $\pm 2.2)$ was significantly lower in relation to T2 $(61.2 \pm 3.3), T 3(62.2 \pm 4.3)$ and T4 (63.7 \pm 4.5$)$ tests. Differences between the intermittent exercise protocols ( $\mathrm{T} 2$ and $\mathrm{T} 4$ ) and the continuous exercise protocols ( $\mathrm{T} 1$ and $\mathrm{T} 3$ ) were also observed. Taken together, the results of the present study suggest that Yo-Yo field can be used in the training program, as a cheaper alternative, aiming the aerobic capacity determination.

Keywords: ${ }^{\top} \mathrm{O}_{2 \text { max }}$ field tests, laboratory tests.

\section{INTRODUÇÃO}

O futebol moderno exige tanto ótimo condicionamento físico como apuradas características físicas ${ }^{(1)}$. A melhora nas capacidades aeróbia e anaeróbia, força muscular, velocidade e coordenação neuromuscular é o principal objetivo de treinamento nesse esporte ${ }^{(2)}$. Os atletas de futebo necessitam se adaptar aos requerimentos do esporte a fim de competirem em alto nível. Nesse sentido, a capacidade aeróbia é uma importante variável para a avaliação do desempenho desses profissionais. Sabe-se que o condicionamento físico e a capacidade aeróbia, em particular, dependem de três importantes elementos: a) consumo máximo de oxigênio; b) limiar anaeróbio; e c) economia de corrida.

Durante os 90 minutos de uma partida de futebol, a contribuição do metabolismo aeróbio aproxima-se de $90 \%$ do total de energia despendida nesse período. Durante esse tempo, os atletas correm cerca de $10 \mathrm{~km}$ de distância em uma intensidade próxima ao limiar anaeróbio, ou a 80-90\% da frequência cardíaca máxima. Estudo recente mostrou que o aumento de $11 \%$ no $\dot{\mathrm{V}}^{\mathrm{T}} \mathrm{O}_{2 \text { máx }}$ elevou em $5 \%$ a distância percorrida em uma partida oficial (aproximadamente 1800 metros) ${ }^{(3)}$.
O futebol inclui, nas suas peculiaridades, alta intensidade e sobrecargas intermitentes de exercício, os quais estimulam as vias metabólicas da glicólise anaeróbia. A contribuição desse metabolismo energético para com o futebol tem sido examinada em vários estudos, principalmente por meio da determinação da concentração de lactato sanguíneo(3).

As respostas à atividade intermitente são caracterizadas por altos níveis de exigência fisiológica se comparadas aos exercícios contínuos realizados em uma mesma carga de trabalho ou exercício.

A capacidade aeróbia do atleta de futebol é rotineiramente avaliada por meio de vários testes de campo e laboratoriais. A maioria desses testes é executada utilizando-se protocolos baseados em tipos de exercícios contínuos. No entanto, um teste é confiável e efetivo quando o mesmo possui caráter específico no que diz respeito aos exercícios aplicados ${ }^{(4)}$. Nesse sentido, muitas equipes vem utilizando o Yo-yo teste de campo na prática diária do futebo|(5).

As respostas fisiológicas ao exercício intermitente tem sido também comparadas àquelas geradas pelo exercício contínuo para uma mesma intensidade média de trabalho, com o intuito de verificar possíveis 
diferenças entre esses dois tipos de atividade. A comparação entre esses dois tipos de exercício, com características distintas, é de extrema relevância, pois ambos são usados no treinamento de futebol com o objetivo de facilitar adaptações fisiológicas e potencializar a melhora do desempenho. Exercício intermitente realizado em uma mesma intensidade média de trabalho, em relação ao exercício contínuo, mostrou estar associado aos efeitos positivos das repostas fisiológicas ${ }^{(6)}$. Por outro lado, outros pesquisadores não obtiveram sucesso em identificar diferenças no custo energético entre os exercícios intermitente e contínuo(7).

Recentemente, foi demonstrado que é possível determinar o $\mathrm{V}_{2 \operatorname{máx}}$ durante teste específico de campo no futebol utilizando, para esse fim, analisador de gás portáti|(1).

São escassas na literatura informações relevantes que avaliem as semelhanças e/ou diferenças fisiológicas entre os exercícios contínuos e intervalados específicos para o futebol, para uma mesma intensidade média de trabalho, utilizando protocolos de campo e laboratoriais.

\section{OBJETIVO}

O presente estudo comparou a aplicação de dois testes específicos de campo (Yo-yo teste contínuo de endurance e Yo-yo teste intermitente de endurance) com dois testes laboratoriais realizados no ergômetro esteira rolante (contínuo e intermitente) na avaliação do consumo máximo de oxigênio $\left({ }^{\mathbb{W}} \mathrm{O}_{2 \text { máx }}\right)$ de atletas de futebol profissionais.

\section{MATERIAL E MÉTODOS}

\section{Sujeitos}

Participaram do presente estudo 20 atletas de futebol profissional com média de idade de 24,11 \pm 0,8 anos. Todos jogadores eram atletas profissionais de futebol e pertencentes a uma equipe do interior do Estado de São Paulo e que, na época do estudo, disputavam a Primeira Divisão do Campeonato Paulista de 2008. A realização de todos ós experimentos envolvendo seres humanos no presente estudo seguiu as normas da resolução específica do Conselho Nacional de Saúde (no 196/96), incluindo a assinatura de um termo de consentimento informado, a proteção da privacidade dos sujeitos.

Todos os sujeitos eram não fumantês, bem como não utilizavam nenhum tipo de agente ergogênico ou medicação conhecida por afetar, positivamente, as funções cardiorrespirató rias durante o estudo.

\section{Desenho experimental}

Todos os atletas realizaram testes de exercícios máximos, dois no campo e dois no laboratorio, para a avaliação da capacidade aeróbia. Os testes de campo foram efetuados em ambiente externo (grama). Os testes foram aplicados aleatoriamente e o tempo de intervalo entre eles foi de dois dias. 0 desenho experimental do estudo encontra-se na tabela 1.

Tabela 1. Desenho experimental do estudo.

\begin{tabular}{c|c}
\hline \multicolumn{1}{c}{ Testes de campo } \\
\hline T1 & $\begin{array}{l}\text { Yo-yo teste de endurance (método indireto de avaliação } \\
\text { da performance aeróbia) }\end{array}$ \\
\hline T2 & $\begin{array}{l}\text { Yo-yo teste intermitente de endurance (ergoespirometria } \\
\text { direta portátil) }\end{array}$ \\
\hline \multicolumn{1}{c}{ Testes de laboratório } \\
\hline T3 & Protocolo contínuo incremental (ergoespirometria direta) \\
\hline T4 & Protocolo de exercício intermitente (ergoespirometria direta) \\
\hline
\end{tabular}

\section{Medidas antropométricas}

Todos os atletas foram submetidos a exames físicos e antropométricos (incluindo determinação da gordura corporal) por meio de mensurações subcutâneas (compasso específico marca Lafayete Instrument $\mathrm{CO}^{\circledR}$ ) e também efetuação de eletrocardiograma (Excel 106 ${ }^{\circledR}$ ). As características antropométricas dos sujeitos estão contidas na tabela 2.

Tabela 2. Características antropométricas dos sujeitos.

\begin{tabular}{c|c} 
Variáveis & $\begin{array}{c}\text { Média } \pm \\
\text { Desvio padrão }\end{array}$ \\
\hline Estatura $(\mathrm{m})$ & $1,80 \pm 0,1$ \\
\hline Peso corporal $(\mathrm{kg})$ & $70,3 \pm 5,6$ \\
\hline Área de superfície corporal $\left(\mathrm{m}^{2}\right)$ & $1,9 \pm 0,1$ \\
\hline Massa corporal magra $(\mathrm{kg})$ & $59,9 \pm 4,3$ \\
\hline Gordura corpora $(\%)$ & $14,8 \pm 2,6$ \\
\hline
\end{tabular}

\section{Testes de campo}

O Yo-yo teste de endurance (T1) foi usado para avaliar a capacidade de correr continuamente por um longo período de tempo. De acordo com esse teste, objetivo básico para o atleta de futebol é percorrer a distancia de 20 metros (movimentação em "vai e vem") o maior número de vezes possível, até a exaustão(5). Quando o participante cessava a atividade, a urtima velocidade e o total percorrido na mesma eram registrados. Os valores de $\dot{\mathrm{V}} \mathrm{O}_{2 \text { máx }}$ foram estimados de acordo com nograma relativo de distância de corrida versus consumo de oxigênio. Yo-yo teste intermitente de endurance (T2), a exemplo de T1, também foi efetuado para avaliar a capacidade dos atletas de futebol em correr por um longo período de tempo a distância de 20 metros ("vai e vem") até a exaustão; entretanto, havia uma pausa de 5 segundos cada vez que o participante percorria duas séries de 20 metros (2×20). Devido à ausência de um nomograma para esse teste, os atletas correram conectados a um sistema portátil telemétrico de registro de $\dot{\mathbf{V}^{\circ}} \mathrm{O}_{2} \mathrm{e}$ $\mathrm{V}^{\circ} \mathrm{CO}_{2}\left(\right.$ Cosmed $\left.\mathrm{K} 2^{\circledR}\right)$. A frequência cardíaca foi registrada a cada cinco segundos e recuperada posteriormente (Polar Sport Tester ${ }^{\circledR}$ ).

A velocidade inicial para ambos os testes (T1 e T2) foi de $9 \mathrm{~km} / \mathrm{h}$, sendo acrescido $1 \mathrm{~km} / \mathrm{h}$ a cada dois minutos. A manutenção das velocidades se deu por meio de estímulo sonoro digital.

\section{Testes laboratoriais}

Todos os atletas efetuaram dois testes adicionais de exercício máximo em esteira rolante (TM 400 Trackmaster $^{\circledR}$ ) após uma semana do término dos testes de campo. O primeiro teste (T3) utilizou protocolo de exercício contínuo (tabela 3), enquanto que o segundo (T4) usou protocolo de exercício intermitente (tabela 4). $\bigcirc \mathrm{V}_{2 \text { máx }}$ foi mensurado durante os dois testes (T3 e T4) por meio de sistema de analisador de gás breath-to-breath (Cosmed Quark b ${ }^{2 \circledR}$ ).

Tabela 3. Protocolo contínuo em esteira (T3).

\begin{tabular}{c|c|c|c}
\hline Estágio & Velocidade (km/h) & Inclinação (\%) & Tempo (min) \\
\hline 1 & 8,0 & 1,0 & 3 \\
\hline 2 & 10,0 & 1,0 & 3 \\
\hline 3 & 12,0 & 1,0 & 3 \\
\hline 4 & 14,0 & 2,0 & 3 \\
\hline 5 & 16,0 & 2,0 & 3 \\
\hline 6 & 18,0 & 2,0 & 3 \\
\hline 7 & 20,0 & 2,0 & 3 \\
\hline
\end{tabular}


Tabela 4. Protocolo intermitente em esteira (T4).

\begin{tabular}{c|c|c|c}
\hline Estágio & Velocidade (km/h) & Inclinação (\%) & Tempo (min) \\
\hline 1 & 9,0 & 0,0 & 3 \\
\hline 2 & 0,0 & 0,0 & 1 \\
\hline 3 & 9,0 & 2,0 & 1 \\
\hline 4 & 9,0 & 6,0 & 1 \\
\hline 5 & 9,0 & 4,0 & 1 \\
\hline 6 & 0,0 & 0,0 & 1 \\
\hline 7 & 9,0 & 6,0 & 1 \\
\hline 8 & 9,0 & 10,0 & 1 \\
\hline 9 & 9,0 & 8,0 & 1 \\
\hline 10 & 0,0 & 0,0 & 1 \\
\hline 11 & 9,0 & 10,0 & 1 \\
\hline 12 & 9,0 & 14,0 & 1 \\
\hline 13 & 9,0 & 12,0 & 1 \\
\hline 14 & 0,0 & 0,0 & 1 \\
\hline 15 & 12,0 & 10,0 & 1 \\
\hline 16 & 12,0 & 14,0 & 1 \\
\hline 17 & 12,0 & 12,0 & 1 \\
\hline 18 & 0,0 & 0,0 & 15,0 \\
\hline 19 & 12,0 & & 1 \\
\hline & & & 1 \\
\hline
\end{tabular}

\section{Parâmetros em comum verificados}

Além das determinações diretas e indiretas do $\dot{\mathrm{V}}_{2 \text { máx }}$ em T1,T2,T3 e T4, foram verificados outros parâmetros em comum, após detectada a exaustão: registro da frequência cardíaca máxima $\left(F C_{\text {máx }}\right)$; registro tempo de exaustão e concentração de lactato sanguíneo máximo, este último descrito a seguir:

\section{Determinação da concentração de lactato sanguíneo máximo (LSM)}

Amostras de sangue ( $25 \mathrm{LL}$ ) foram coletadas do lóbulo da orelha dos participantes, com o auxílio de capilares de vidro heparinizados, após cinco minutos da detecção da exaustão, a fir de se obter a concentração de lactato sanguíneo máximo (pico) desses sujeitos ${ }^{(8)}$. Os valores de lactato sanguíneo foram obtidos por meio de analisador específico (Lactímetro - Modelo YSI 1500 sport ${ }^{\circledR}$.

\section{ANÁLISE ESTATÍSTICA}

Todos os resultados foram expressos como média \pm desvio padrão. Foi utilizada ANOVA de duas entradas para medidas repetidas a fim de se determinar diferenças significantes entre os quatro testes aplicados no presente estudo (ORIGIN 6.0 $0^{\circledR}$. O nível de significância estatística foi preeestabelecido em

\section{RESULTADOS}

Os valores médios referentes às variáveis analisadas no presente estudo estão contidos na Tabela 5.

O tempo de exaustão (TE) foi significativamente maior nos testes $\mathrm{T} 2$ e T4, os quais tiveram sobrecargas intermitentes, se comparado com T1 e T3. No entanto, o tempo de exercício efetuado foi maior em T4. A duração do tempo de exercício em T3 foi significativamente maior se comparado a $\mathrm{T} 1$.

Em T1, os atletas apresentaram menores magnitudes de $\dot{\mathrm{v}} \mathrm{O}_{2 \text { máx }}$ em comparação aos outros três testes. Ainda, diferença significativa foi também encontrada nos valores de $\dot{\mathrm{V}} \mathrm{O}_{2 \text { máx }}$ entre os testes de caráter intermitente (T2 e T4) e entre os testes de natureza contínua (T1 e T3). A concentração de lactato sanguíneo máximo (LSM) em T2 mostrou-se significativamente elevada em relação a T1 e T3. Não foi observada diferença estatística no LSM entre T2 e T4.

Em relação à frequência cardíaca máxima ( $F C_{\text {máx }}$, não foram encontradas diferenças estatísticas entre os 4 testes (tabela 5).

Tabela 5. Valores médios $(n=20)$ de tempo de exaustão (TE; min), lactato sanguíneo máximo $\left(\mathrm{LSM} ; \mathrm{mmol} / \mathrm{L}^{-1}\right)$, frequência cardíaca máxima $\left(\mathrm{FC}_{\text {máxi }}\right.$ b.min $\left.{ }^{-1}\right)$

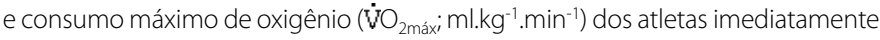
após (com exceção do lactato sanguíneo máximo = após 5 minutos da verificação da exaustão) a detecção da exaustão na realização dos testes T1 (Yo-yo teste de endurance), T2 (Yo-yo teste intermitente de endurance), T3 (protocolo contínuo em esteira) e T4 (protocolo intermitente em esteir

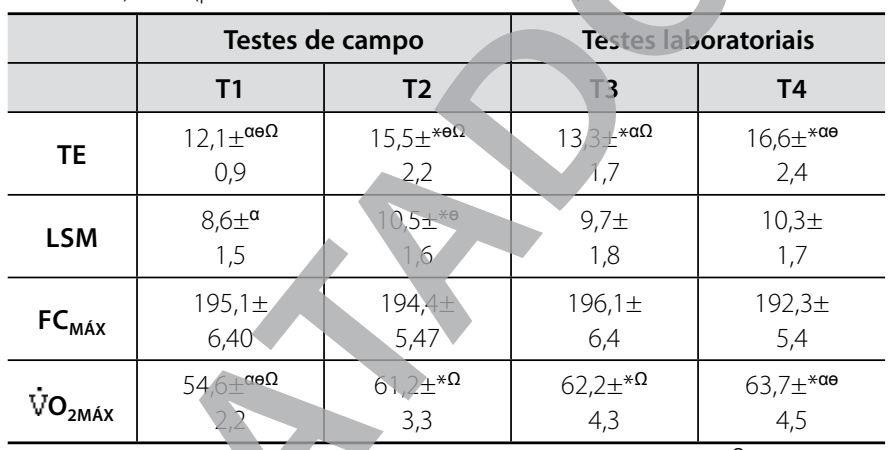

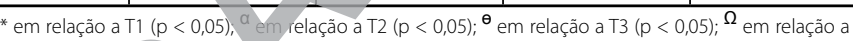
$\mathrm{T} 4 \mathrm{p}(\mathrm{p}<0,05)$.

\section{DISCUSSÃO}

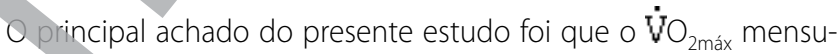
rado nos at letas que realizaram exercício em esteira rolante (T3 e T4) mostrou-se elevado em relação aos testes efetuados no campo (T1 e 12). Tais resultados sugerem que os testes Yo-yo (contínuo e/ou internte), com ou sem a utilização do método telemétrico, subestima os valores de mensuração de $\mathrm{V}_{2 \text { máx }}$ No entanto, estes métodos de mensuração do $\dot{\mathrm{V}}_{2 \text { máx }}$ não devem ter seu uso excluído, uma vez que os mesmos podem ser facilmente aplicados e são componentes importantes do treinamento específico de futebol.

Os dados antropométricos dos atletas do presente estudo foram similares aos encontrados em estudos referentes ao futebo ${ }^{(9)}$. Estudos antropométricos em atletas de futebol tem mostrado que altura e peso corporais são fatores importantes na performance desses jogadores ${ }^{(10)}$. Sabe-se que a alta porcentagem de gordura corporal reduz o desempenho.

A capacidade aeróbia dos atletas do presente estudo, estimada diretamente (ergoespirômetro portátil) por meio do Yo-yo teste intermitente de endurance, está de acordo com os valores que vem sendo reportados para jogadores de elite usando o mesmo método ${ }^{(11)}$. Valores similares de $\mathrm{V}_{2 \operatorname{máx}}$ também foram observados em atletas de elite adultos e submetidos ao mesmo protocolo de teste ${ }^{(12)}$.

O Yo-yo teste intermitente de endurance tem provado ser uma mensuração válida do desempenho físico no futebol, possuindo alta reprodutibilidade. Os resultados obtidos por Krustrup et al. ${ }^{(4)}$ estão de acordo com outros e suportam a ideia de que o ergoespirômetro telemétrico portátil caracteriza-se por ser um método confiável de determinação da capacidade aeróbia de atletas no campo $^{(13)}$. É destacado que este método pode contribuir efetivamente para a criação de melhores planos de treino de futebol e, por consequência, elevar o nível de performance de seus praticantes. Helgerud et al.(3) mostraram que o treinamento intervalado (90-95\% da $F C_{\text {máx }}$ - quatro períodos de quatro minutos separados por três minutos de repouso ativo $\left(70 \%\right.$ da $\left.\mathrm{FC}_{\text {máx }}\right)$, duas vezes/semana - durante nove semanas - elevou o $\dot{\mathrm{V}}_{2 \text { máx }}$ em $11 \%$ de atletas de futebol, além de aumentar em 100\% o número de sprints ao longo da partida. 
Stolen et al. ${ }^{(14)}$, em seu artigo de revisão sobre atualidades fisiológicas do futebol, recomendam o teste específico de campo Yo-yo intermitente de endurance para equipes que não possuem fácil acesso aos laboratórios de mensurações diretas do $\overline{\mathrm{V}}_{2 \text { máx’ }}$ com o intuito de avaliar a performance de atletas de futebol.

Os baixos valores de $\dot{\mathrm{V}} \mathrm{O}_{2 \text { máx }}$ observados no Yo-yo teste contínuo de endurance comparados ao Yo-yo teste intermitente, no presente estudo, podem ser devidos a vários fatores, tais como a mudança de direção no movimento de "vai e vem" bem como intensidade de corrida instável. Outra possível causa é o efeito da aceleração imediatamente após o estímulo "sinal-virada". Ainda, encorajamento e competição entre os jogadores durante o teste podem ter contribuído com esses resultados de $\mathrm{V}^{\mathrm{V}} \mathrm{O}_{2 \text { máx }}{ }^{(15)}$.

Os valores de $\dot{\mathrm{V}} \mathrm{O}_{2 \text { máx }}$ obtidos em ambos os testes de laboratório (T3 e T4) mostraram-se elevados em relação aos valores observados nos testes de campo (T1 e T2). Essa diferença pode ser explicada, pelo menos em parte, pelas diferentes condições de corrida, considerando que as viradas do movimento de "vai e vem" no campo podem constituir um fator de diminuição dos valores de ${ }^{\mathrm{V}} \mathrm{O}_{2 m a ́ x}$. Essa ideia é também suportada, no que diz respeito às diferentes condições de corrida, pelos protocolos de sobrecargas intermitentes, sendo que os mesmos demonstram maiores valores de $\dot{\mathrm{V}}^{\mathrm{V}} \mathrm{O}_{2 \text { máx }}$ em comparação aos protocolos de exercícios contínuos ${ }^{(16)}$.

Embora os valores de $\dot{\mathrm{V}} \mathrm{O}_{2 \text { máx }}$ do presente estudo mostrem diferenças entre os grupos, os mesmos corroboram magnitudes comumente observadas em atletas de futebol profissional de outros estudos, sendo estes reportados entre 55 e $68 \mathrm{ml} . \mathrm{kg}^{-1} \cdot \mathrm{min}^{-1}$. Estes valores são classificados de moderado a alto e são similares aos de outros esportes coletivos $^{(17)}$, porém estão significativamente abaixo de esportes estritamente caracterizados pela performance aeróbia $\left(90 \mathrm{ml}^{\mathrm{kg}} \mathrm{kg}^{-1} \cdot \mathrm{min}^{-1}\right)^{(18)}$. Valores individuais acima de $70 \mathrm{ml} . \mathrm{kg}^{-1} \cdot \mathrm{min}^{-1}$ tem sido encontrados em atletas de futebol profissional(3).

Os atletas do presente estudo apresentaram maiores valores de $\dot{\mathrm{V}} \mathrm{O}_{2 \text { máx }}$ durante o teste intermitente de laboratório. Isto pode ser explicado pelo fato de que em protocolos intermitentes com essas características (Tabela 4), a fase de recuperação contribui, de maneira efetiva, para a alta produção de trabalho muscular ${ }^{(19)}$.

Durante os testes de campo e laboratoriais não foi observada diferença significativa em relação às magnitudes da FC náx. Tal achado nos permite afirmar que todos os atletas atingiram seus máximos esforços quando submetidos aos diferentes protocol

O alto nível de lactato sanguíneo máximo (lSM) observado nos testes T1, T2, T3 e T4 também é critério de alcance do $\mathrm{W}_{2 \text { máx }}$ e demonstra o uso da produção de energla anaeróbia durante os testes.

\section{REFERÊNCIAS}

1. Hoff J, Wisloff U, Engen LC, Kemi OJ,Helgerud J. Soccer specific aerobic endurance training. Br J Sports Med 2002;36:218-21.

2. Sendelides T, Kouidi E, Metaxas, Koutlianos N, Deligiannis A. Cardiorespiratory adaptations in soccer players. Hung Rev Sports Med 2003:44:141-51.

3. Helgerud J, Engen LC Wisloff U, Hoff J. Aerobic endurance training improves soccer performance. Med Sci Sports Exerc

4. Krustrup P, Mohr M, Amstrup T, Rysgaard T, Johansen J, Steenberg A, et al. The Yo-Yo intermittent recovery test: physiological response, reliability and validity. Med Sci Sports Exerc 2003;35:697-705.

5. Bangsbo J. The physiology of soccer: with special reference to intense intermittent exercise. Acta Physiol Scand 1996;15(619):1-156.

6. Edwards GE, Ekelund LG, Harris RC, Hesses CM, Hultman E. Cardiorespiratory and metabolic costs of continuous and intermittent exercise in man. J Physiol 1973;234:481-97.

7. Astrand PO, Rodahl K. Textbook of work physiology. 2 nd rev. ed. New York: Mcraw-Hill, 1977.

8. Tegtbur U, Busse MW, Braumann KM. Estimation of individual equilibrium between production and catabolism during exercise. Med Sci Sports Exerc 1993;25:620-7.

9. Tiryaki G, Tuncel F, Yamaner F, Agaoglu SA, Gumubdad H, Acar MF. Comparison of the physiological characteristics of the first, second and third league Turkish soccer players. In: Science and Football III. Reilly T, Bangsbo J, Hughes M, eds. London: E \& FN Spon, 1997;32-6.

10. Reilly T, Bangsbo J, Franks A. Anthropometric and physiological predispositions for elite soccer. J Sports Sci 2000;18:669-83.
Os valores mais elevados, os quais foram obtidos durante os testes intervalados (T2 e T4), são resultantes das adaptações anaeróbias a esforços de alta intensidade dessa natureza. Ao final do Yo-yo teste de endurance contínuo (T1), os jogadores de futebol do presente estudo demonstraram, de forma significativa, menor concentração de lactato sanguíneo quando comparada às concentrações obtidas tanto em T2 (campo) como em T3 e T4 (laboratoriais). Tal ocorrência pode ser devida à baixa carga máxima de exercício que os atletas suportaram no último estágio do teste (exaustão). A redução da velocidade máxima pode ser ocasionada pela fadiga muscular local, causada pela corrida (movimentação em "vai e vem") durante os testes de campoem comparação aos testes conduzidos em laboratório, sendo que esses últimos apresentam, tanto no exercício contínuo como no progressivo, aumento progressivo da carga de trabalho(20), como descrito na metodologia do presente estudo (Tabelas 3 e 4).

A taxa de remoção de lactato sanguíneo no futebol depende diretamente da concentração desse substrato, tipo de atividade no período de recuperação e capacidade aeróbia. Foi relatado que quanto maior a concentração de lactato sanguíneo, maior é a sua taxa de remoção(5). É importante ressaltar que jogadores com elevado $\dot{\mathrm{V}}_{2 \text { máx }}$ apresentam menor concentração de lactato sanguíneo durante, principalmente, o segundo tempo, devido a uma melhora na recuperação de intensidades intermitentes atraves. a) do aumento da resposta aeróbia; b) da melhora na remoção de lactato; e c) da melhora na regeneração de fosfocreatina ${ }^{(21)}$. Em uma situação real de jogo, na qual a intensidade esteja em torno de $70 \%$ da FC máxı por exemplo, a remoção de lactato nesses jogadores mais bem condicionados aerobiamente será mais eficiente.

\section{CONCLUSAO}

A partir dos resultados obtidos no presente estudo e da discussão apresentada, podemos concluir que:

a determinação do $\dot{\mathbf{V}}_{2 \text { máx }}$ em atletas de futebol utilizando protocoos de teste em esteira rolante parece ser, agudamente, o método mais indiçado se comparado aos Yo-yo testes. No entanto, a determinação do $\mathrm{V}_{2} \mathrm{O}_{2 \text { áx }}$ por meio do método de telemetria no campo é também confiável para a avaliação da capacidade aeróbia desses atletas;

os Yo-yo testes de campo são considerados ferramenta de prática e fácil aplicação para treinadores que desejam planejar exercícios específicos para o desenvolvimento da capacidade aeróbia de seus atletas, sendo eles de elite ou não.

Todos os autores declararam não haver qualquer potencial conflito de interesses referente a este artigo.

11. Nicholas CW, Nuttall FE, Williams C. The longh-borough intermittent shuttle run test: a field test that simulates the activity pattern of soccer. J Sports Sci 2000;18: 97-104.

12. Metaxas T, Koutlianos N, Sedelides T, Kouidi E. Evaluation o the level of aerobic capacity in pubertal soccer players in the field test. Health Sports Perform 2001;4:316-22.

13. Crandal C, Taylor S, Raven P. Evaluation of the Cosmed K2 portable telemetric oxygen uptake analyzer Med Sci Sports Exerc 1994;26:108-11.

14. Stolen T, Chamari K, Castagna C, Wisloff U. Physiology of soccer - an update. Sports Med 2005;35:501-36.

15. Moffatt $R$, Chitwood $L$, Biggerstaff $K$. The influence of verbal encouragement during assessment of maximal oxygen uptake. J Sports Med Phys Fitness 1994;34:45-9.

16. Duncan GE, Howley ET, Johnson BN. Applicability of $\mathrm{VO}_{2 \max }$ criteria: discontinuous versus continuous protocols. Med Sci Sports Exerc 1997;29:272-8.

17. Ekblom B. Applied physiology of soccer. Sports Med 1986;45:50-60.

18. Hoff J, Helgerud J. Endurance and strength training for soccer players. Physiological considerations. Sports Med 2004;34:165-80

19. Wisloff U, Helgerud J, Hoff J. Strength and endurance of elite soccer players. Med Sci Sports Exerc 1998:30:462-7.

20. Drust B, Cable NT, Reilly T. Investigation of the effect of pre-cooling on the physiological responses to soccer-specific intermittent exercise. Eur J Appl Physiol 2000;81:11-7.

21. Tomlin DL, Wenger HA. The relationship between aerobic fitness and recovery from high intensity exercise. Sports Med 2001;31:1-11. 